\title{
Asymptotic Properties of Self-Normalized Linear Processes with Long Memory
}

\author{
Magda Peligrad 41 and Hailin Sang $^{b}$ \\ ${ }^{a}$ Department of Mathematical Sciences, University of Cincinnati, PO Box \\ 210025, Cincinnati, OH 45221-0025, USA. E-mail address: peligrm@ucmail.uc.edu \\ ${ }^{b}$ National Institute of Statistical Sciences, PO Box 14006, Research Triangle \\ Park, NC 27709, USA. E-mail address: sang@niss.org
}

Mathematical Subject Classification (2010): 60F05, 60F17, 60G10, 60G22.

Key words and phrases: linear processes, long memory, invariance principle, fractional Brownian motion, domain of normal attraction, fractionally integrated processes, unit root.

\begin{abstract}
In this paper we study the convergence to fractional Brownian motion for long memory time series having independent innovations with infinite second moment. For the sake of applications we derive the self-normalized version of this theorem. The study is motivated by models arising in economical applications where often the linear processes have long memory, and the innovations have heavy tails.
\end{abstract}

\section{Introduction and notations}

In this paper we study the asymptotic properties of a causal linear process

$$
X_{k}=\sum_{i \geq 0} a_{i} \varepsilon_{k-i}
$$

when the i.i.d. innovations $\left\{\varepsilon, \varepsilon_{n} ; n \in \mathbb{Z}\right\}$ have infinite variance and $\left\{a_{i} ; i \geq 0\right\}$ is a sequence of real constants such that $X_{k}$ is well defined. More precisely, everywhere in the paper, we assume that the innovations are centered and in the domain of attraction of a normal law. This means that the variables are independent, identically distributed,

$$
E \varepsilon=0
$$

\footnotetext{
${ }^{1}$ Supported in part by a Charles Phelps Taft Memorial Fund grant and NSA grant H9823009-1-0005.
} 
and

$$
l(x)=E \varepsilon^{2} I(|\varepsilon| \leq x) \text { is a slowly varying function at } \infty .
$$

We say that $h(t)$, defined for $t \geq 0$, is slowly varying if it is positive and measurable on $[A, \infty)$, for some $A>0$, and if for any $\lambda>0$, we have $\lim _{x \rightarrow \infty} h(\lambda x) / h(x)=$ 1 (Seneta, 1970, Definition 1.1).

We define

$$
S_{n}=\sum_{i=1}^{n} X_{i} .
$$

The central limit theorem for $S_{n}$ with i.i.d. innovations and infinite variance when $\sum_{i>0}\left|a_{i}\right|<\infty$ was studied by many authors. We mention among them, Knight (1991), Mikosch et al (1995), Wu (2003). For this case the central limit theorem was obtained under a normalization that is regularly varying with exponent $1 / 2$.

The purpose of this paper is to investigate the central limit theorem in its functional form for the case when

$$
a_{n}=n^{-\alpha} L(n), \text { where } 1 / 2<\alpha<1, n \geq 1
$$

and $L(n)$ is a slowly varying function at $\infty$ in the strong sense (i.e. there is a slowly varying function $h(t)$ such that $L(n)=h(n))$. Notice that, by the definition of slowly varying function, the coefficients $a_{n}$ are positive for $n$ sufficiently large. We shall obtain convergence in distribution under a normalization that is regularly varying with exponent $3 / 2-\alpha$ which is strictly larger than $1 / 2$. This is the reason why the time series we consider has long memory.

To give an example of a linear process of this type we mention the fractionally integrated processes since they play an important role in financial time series modeling and they are widely studied. Such processes are defined for $0<d<$ $1 / 2$ by

$$
X_{k}=(1-B)^{-d} \varepsilon_{k}=\sum_{i \geq 0} a_{i} \varepsilon_{k-i} \text { with } a_{i}=\frac{\Gamma(i+d)}{\Gamma(d) \Gamma(i+1)},
$$

where $B$ is the backward shift operator, $B \varepsilon_{k}=\varepsilon_{k-1}$. For this example, by the well known fact that for any real $x, \lim _{n \rightarrow \infty} \Gamma(n+x) / n^{x} \Gamma(n)=1$, we have $\lim _{n \rightarrow \infty} a_{n} / n^{d-1}=1 / \Gamma(d)$.

The CLT in its functional form was intensively studied for the case of i.i.d. innovations with finite second moment. We refer to Davydov (1970), Taqqu (1975), Phillips and Solo (1992), Wang et al (2003), Wu and Min (2005), Dedecker et al (2009), among others. Invariance principles (or functional central limit theorems) play an important role in econometrics and statistics. For example, to obtain asymptotic distributions of unit-root test statistics, researchers have applied invariance principles of various forms; see Phillips (1987) and Wu (2006).

We shall derive here the central limit theorem and its functional form, i.e. convergence to fractional Brownian motion, for the case when the innovations 
are in the domain of attraction of the normal distribution and the constants satisfy (4). The normalizer in this theorem depends on the slowly varying function $l(x)$ that is in general unknown. To make our results easily applicable we also study the central limit theorem in its self-normalized form.

The self-normalized CLT for sums of independent identically distributed random variables was treated in the paper by Giné et al (1997). The case of selfnormalized sums in the domain of attraction of other stable laws was considered by Chistyakov and Götze (2004). A systematic treatment of self-normalized limit theory under independence assumption is given in the book by de la Peña et al (2009). The self-normalized version of the functional central limit theorem for this case, was treated in Csörgö et al (2003). Kulik (2006) studied the selfnormalized functional CLT when $\sum_{i>0}\left|a_{i}\right|<\infty$. We shall consider the long memory case when coefficients satisfy (4).

Our paper is organized in the following way: Section 2 contains the definitions and the results; the proofs are given in section 3. For convenience, in the Appendix, we give some auxiliary results and we also mention some known facts needed for the proofs.

In this paper we shall use the following notations: a double indexed sequence with indexes $n$ and $i$ will be denoted by $a_{n i}$ when no confusion is possible, and sometimes by $a_{n, i}$; we use the notation $a_{n} \sim b_{n}$ instead of $a_{n} / b_{n} \rightarrow 1$; for positive sequences, the notation $a_{n} \ll b_{n}$ replaces Vinogradov symbol $O$ and it means that $a_{n} / b_{n}$ is bounded; $a_{n}=o\left(b_{n}\right)$ stays for $a_{n} / b_{n} \rightarrow 0 ;[x]$ denotes the integer part of $x$; the notation $\Rightarrow$ is used for weak convergence, and $\stackrel{P}{\rightarrow}$ denotes convergence in probability. By $\operatorname{var}(X)$ we denote the variance of the random variable $X$ and by $\operatorname{cov}(X, Y)$ the covariance of $X$ and $Y$. The weak convergence to a constant means convergence in probability. We denote by $D[0,1]$ the space of all functions on $[0,1]$ which have left-hand limits and are continuous from the right. $N(0,1)$ denotes a standard normal random variable.

\section{Results}

To introduce our results we define a normalizing sequence in the following way. Recall (3) and (44). Let $b=\inf \{x \geq 1: l(x)>0\}$, define

$$
\eta_{j}=\inf \left\{s: s \geq b+1, \frac{l(s)}{s^{2}} \leq \frac{1}{j}\right\}, \quad j=1,2, \cdots
$$

and set

$$
B_{n}^{2}:=c_{\alpha} l_{n} n^{3-2 \alpha} L^{2}(n) \text { with } l_{n}=l\left(\eta_{n}\right)
$$

where

$$
c_{\alpha}=\left\{\int_{0}^{\infty}\left[x^{1-\alpha}-\max (x-1,0)^{1-\alpha}\right]^{2} d x\right\} /(1-\alpha)^{2} .
$$

Theorem 2.1 Define $\left\{X_{n} ; n \geq 1\right\}$ by (1) and the random element $W_{n}(t)=$ $S_{[n t]} / B_{n}$ on the space $D[0,1]$. Assume conditions (2), (3) and (4) are satisfied. Then, $W_{n}(t)$ converges weakly on the space $D[0,1]$ endowed with Skorohod 
topology to the fractional Brownian motion $W_{H}$ with Hurst index $H=3 / 2-\alpha$. In particular, for $t=1$, we have that $S_{n} / B_{n}$ converges in distribution to a standard normal variable.

Remark 2.1 In a forthcoming paper the authors treat the central limit theorem for the situation when $B_{n}^{2}$ is not necessarily regularly varying. However, for that situation the convergence to the fractional Brownian motion might fail. As a matter of fact, in the context of Theorem 2.1 a necessary condition for the convergence to the fractional Brownian motion $W_{H}$ with Hurst index $H=\beta$ is the representation $B_{n}^{2}=n^{2 \beta} h(x)$ for a function $h(x)$ that is slowly varying at infinity (see Lamperti, 1962).

For successfully applying this theorem we have to know $l_{n}$ that depends on the distribution of $\varepsilon$. This can be avoided by constructing a selfnormalizer. Denote $\sum_{i=0}^{\infty} a_{i}^{2}=A^{2}$. Our result is:

Theorem 2.2 Under the same conditions as in Theorem 2.1 we have

$$
\frac{1}{n l_{n}} \sum_{i=1}^{n} X_{i}^{2} \stackrel{P}{\rightarrow} A^{2}
$$

and therefore

$$
\frac{S_{[n t]}}{n a_{n} \sqrt{\sum_{i=1}^{n} X_{i}^{2}}} \Rightarrow \frac{\sqrt{c_{\alpha}}}{A} W_{H}(t) .
$$

In particular

$$
\frac{S_{n}}{n a_{n} \sqrt{\sum_{i=1}^{n} X_{i}^{2}}} \Rightarrow N\left(0, \frac{c_{\alpha}}{A^{2}}\right)
$$

\section{Application to unit root testing}

Invariance principles play an important role in characterizing the limit distribution of various statistics arising from the inference in economic time series.

Let us consider a stochastic process generated according to

$$
Y_{n}=\rho Y_{n-1}+X_{n} \text { for } n \geq 1
$$

where $Y_{0}=0$ and $\left(X_{n}\right)_{n \geq 1}$ is a stationary sequence and $\rho$ is a constant. Denote the ordinary least squares (OLS) estimator of $\rho$ by

$$
\hat{\rho}_{n}=\sum_{k=1}^{n} Y_{k} Y_{k-1} / \sum_{k=1}^{n} Y_{k-1}^{2} .
$$

To test $\rho=1$ against $\rho<1$, a key step is to derive the limit distribution of the well-known Dickey-Fuller (DF) test statistic (Dickey and Fuller 1979, 1981):

$$
\hat{\rho}_{n}-1=\sum_{k=1}^{n} Y_{k-1}\left(Y_{k}-Y_{k-1}\right) / \sum_{k=1}^{n} Y_{k-1}^{2} .
$$


As shown by Phillips (1987), under the null hypothesis $\rho=1$, the asymptotic properties of the DF test statistic rely heavily on the invariance principles. This problem was widely studied under various assumptions on the sequence $X_{n}$. Among them Sowell (1990) and $\mathrm{Wu}$ (2006) considered the unit root testing problem for long-memory processes. By combining our Theorems 2.1 and 2.2 with arguments similar to Phillips (1987), we can formulate the following result obtained for variables that do not necessarily have finite second moment.

Proposition 3.1 Assume that $\left(X_{n}\right)_{n \geq 1}$ is as in Theorem [2.1. Then the following results hold

$$
\begin{aligned}
& \frac{\sum_{k=1}^{n} Y_{k-1}^{2}}{n^{3} a_{n}^{2} \sum_{i=1}^{n} X_{i}^{2}} \Rightarrow \frac{c_{\alpha}}{A^{2}} \int_{0}^{1} W_{H}^{2}(t) d t . \\
& \frac{\sum_{k=1}^{n} Y_{k-1}\left(Y_{k}-Y_{k-1}\right)}{n^{2} a_{n}^{2} \sum_{i=1}^{n} X_{i}^{2}} \Rightarrow \frac{c_{\alpha} W_{H}^{2}(1)}{2 A^{2}} . \\
& n\left(\hat{\rho}_{n}-1\right) \Rightarrow \frac{W_{H}^{2}(1) / 2}{\int_{0}^{1} W_{H}^{2}(t) d t} .
\end{aligned}
$$

The proof of this proposition requires only to make obvious changes in the proofs of (A1) and (A2) on page 296 in Phillips (1987), and it is left to the reader.

\section{Proofs}

\subsection{Proof of theorem 2.1}

In order to prove the central limit theorem in its functional form, i.e. the weak convergence of $S_{[n t]} / B_{n}$ on the space $D[0,1]$ to the fractional Brownian motion $W_{H}$ with Hurst index $H=3 / 2-\alpha$, we shall first reduce the problem to truncated random variables. For the truncated process we establish tightness on $D[0,1]$ and the convergence of finite dimensional distributions.

Without the loss of generality, in the rest of the paper, we assume for convenience $a_{0}=0$ in definition (1).

We shall divide the proof in several steps:

Step 1. Existence.

To show that $X_{1}$ is well defined we use stationarity and Lemma 5.2 from the Appendix. First of all we have

$$
\sum_{i=1}^{\infty} P\left(\left|a_{i} \varepsilon_{1-i}\right|>1\right)=\sum_{i=1}^{\infty} P\left(|\varepsilon|>\left|a_{i}\right|^{-1}\right)=\sum_{i=1}^{\infty} a_{i}^{2} o\left(l\left(\left|a_{i}\right|^{-1}\right)\right) .
$$

Then, by taking into account that (2) implies $E \varepsilon I\left(|\varepsilon| \leq\left|a_{i}\right|^{-1}\right)=-E \varepsilon I(|\varepsilon|>$ $\left.\left|a_{i}\right|^{-1}\right)$,

$$
\sum_{i=1}^{\infty}\left|E a_{i} \varepsilon_{1-i} I\left(\left|a_{i} \varepsilon_{1-i}\right| \leq 1\right)\right| \leq \sum_{i=1}^{\infty}\left|a_{i}\right| E|\varepsilon| I\left(|\varepsilon|>\left|a_{i}\right|^{-1}\right)=\sum_{i=1}^{\infty} a_{i}^{2} o\left(l\left(\left|a_{i}\right|^{-1}\right)\right)
$$


and

$$
\sum_{i=1}^{\infty} E a_{i}^{2} \varepsilon_{1-i}^{2} I\left(\left|a_{i} \varepsilon_{1-i}\right| \leq 1\right)=\sum_{i=1}^{\infty} a_{i}^{2} E \varepsilon^{2} I\left(|\varepsilon| \leq\left|a_{i}\right|^{-1}\right)=\sum_{i=1}^{\infty} a_{i}^{2} l\left(\left|a_{i}\right|^{-1}\right) .
$$

Notice that

$$
\sum_{i=1}^{\infty} a_{i}^{2} l\left(\left|a_{i}\right|^{-1}\right)=\sum_{i=1}^{\infty} i^{-2 \alpha} L^{2}(i) l\left(i^{2 \alpha} L^{-2}(i)\right)<\infty,
$$

since $1 / 2<\alpha<1$ and $L^{2}(i) l\left(i^{2 \alpha} L^{-2}(i)\right)$ is a slowly varying function at $\infty$. The existence in the almost sure sense follows by combining these arguments with the three series theorem.

\section{Step 2. Truncation.}

For the case when $E \varepsilon^{2}=\infty$, which is relevant to our paper, the truncation is necessary. The challenge is to find a suitable level of truncation. For any integer $1 \leq k \leq n$ define

$$
X_{n k}^{\prime}=\sum_{i=1}^{\infty} a_{i} \varepsilon_{k-i} I\left(\left|\varepsilon_{k-i}\right| \leq \eta_{n-k+i}\right) \text { and } S_{n}^{\prime}=\sum_{k=1}^{n} X_{n k}^{\prime} .
$$

This definition has the advantage that $S_{n}^{\prime}$ can be expressed as a simple sum of a linear process of an array of independent variables. For every $m \geq 1$ we denote

$$
b_{m}=a_{1}+\ldots+a_{m},
$$

and then we introduce the coefficients

$$
\begin{aligned}
& b_{n j}=b_{j}=a_{1}+\ldots+a_{j} \text { for } j<n \\
& b_{n j}=b_{j}-b_{j-n}=a_{j-n+1}+\ldots+a_{j} \text { for } j \geq n .
\end{aligned}
$$

With this notation and recalling definition (6), by changing the order of summation,

$$
S_{n}^{\prime}=\sum_{i \geq 1} b_{n i} \varepsilon_{n-i} I\left(\left|\varepsilon_{n-i}\right| \leq \eta_{i}\right) .
$$

We shall reduce next, the study of limiting distribution of $S_{n} / B_{n}$ to the sequence $S_{n}^{\prime} / B_{n}$. It is enough to show that

$$
\frac{1}{B_{n}} E\left|S_{n}-S_{n}^{\prime}\right| \rightarrow 0
$$

To see this we use the fact that by Lemma 5.2 stated in the Appendix

$$
E|\varepsilon| I\left(|\varepsilon|>\eta_{i}\right)=o\left(\eta_{i}^{-1} l_{i}\right) .
$$

We also know that

$$
\eta_{n}^{2} \sim n l_{n}
$$


(see for instance relation 13 in Csörgö et al, 2003). Then, by the triangle inequality and relation (40) of Lemma 5.4 from the Appendix applied with $p=1$, we obtain

$$
\begin{gathered}
E\left|S_{n}-S_{n}^{\prime}\right| \leq \sum_{i \geq 1}\left|b_{n i}\right| E|\varepsilon| I\left(|\varepsilon|>\eta_{i}\right)=\sum_{i \geq 1}\left|b_{n i}\right| o\left(\eta_{i}^{-1} l_{i}\right) \\
=\sum_{i \geq 1}\left|b_{n i}\right| o\left(i^{-1 / 2} l_{i}^{1 / 2}\right)=o\left(n^{3 / 2-\alpha} l_{n}^{1 / 2} L(n)\right)=o\left(B_{n}\right)
\end{gathered}
$$

and so (14) is established.

Step 3. Central limit theorem.

To make the proof more transparent we shall present first the central limit theorem for $S_{n} / B_{n}$. By the Step 2 it is enough to find the limiting distribution of $S_{n}^{\prime} / B_{n}$. We start by noticing that by (16) and the fact that the variables are centered we have

$$
\left|E S_{n}^{\prime}\right|=\left|E\left(S_{n}-S_{n}^{\prime}\right)\right|=o\left(B_{n}\right) .
$$

One of the consequences of this observation is that $S_{n}^{\prime} / B_{n}$ has the same limiting distribution as $\left(S_{n}^{\prime}-E S_{n}^{\prime}\right) / B_{n}$. Furthermore,

$$
\operatorname{var}\left(\frac{S_{n}^{\prime}}{B_{n}}\right)=\frac{1}{B_{n}^{2}} \sum_{i \geq 1} b_{n i}^{2} l_{i}-\frac{1}{B_{n}^{2}}\left(E S_{n}^{\prime}\right)^{2} \rightarrow 1
$$

by relation (39) in Lemma 5.4 and (17).

Moreover, by the point 1 in Lemma 5.4 for $k_{n}=n^{4 /(2 \alpha-1)}$

$$
\begin{aligned}
\operatorname{var}\left(\sum _ { i \geq k _ { n } } b _ { n i } \varepsilon _ { n - i } I \left(\left|\varepsilon_{n-i}\right|\right.\right. & \left.\left.\leq \eta_{i}\right)\right) \ll \sum_{i \geq k_{n}} n^{2}(i-n)^{-2 \alpha} L^{2}(i) l_{i} \\
& =o(1) \text { as } n \rightarrow \infty .
\end{aligned}
$$

Then, by Theorem 4.1 in Billingsley (1968), for proving the central limit theorem it is enough to verify Lyapunov's condition for $B_{n}^{-1}\left(\bar{S}_{n}^{\prime}-E \bar{S}_{n}^{\prime}\right)$ where

$$
\bar{S}_{n}^{\prime}=\sum_{i=1}^{k_{n}} b_{n i} \varepsilon_{n-i} I\left(\left|\varepsilon_{n-i}\right| \leq \eta_{i}\right) .
$$

Clearly, by (18), $\operatorname{var}\left(\bar{S}_{n}^{\prime} / B_{n}\right) \rightarrow 1$. In the estimate below we use the point 4 of Lemma 5.2 along with (15), followed by relation (40) of Lemma 5.4 applied with $p=3$ and the fact that $B_{n} \rightarrow \infty$ to get:

$$
\begin{gathered}
\sum_{j=1}^{k_{n}}\left|b_{n j}\right|^{3} E\left|\varepsilon^{\prime}-E \varepsilon^{\prime}\right|^{3} \leq 8 \sum_{j=1}^{k_{n}}\left|b_{n j}\right|^{3} E|\varepsilon|^{3} I\left(|\varepsilon| \leq \eta_{j}\right) \\
=\sum_{j=1}^{k_{n}}\left|b_{n j}\right|^{3} \eta_{j} o\left(l_{j}\right) \leq \sum_{j=1}^{\infty}\left|b_{n j}\right|^{3} j^{1 / 2} o\left(l_{j}^{3 / 2}\right)=o\left(n^{3(3 / 2-\alpha)} l_{n}^{3 / 2} L^{3}(n)\right)=o\left(B_{n}^{3}\right) .
\end{gathered}
$$


By Lyapunov's central limit theorem and the above considerations, $S_{n} / B_{n}$ converges to $N(0,1)$ in distribution.

Step 4. Preliminary considerations for the convergence to fractional Brownian motion.

For $n \geq 1$ fixed we implement the same level of truncation as before and construct $\left\{X_{n j}^{\prime} ; 1 \leq j \leq n\right\}$ by definition (10). Then we introduce the processes

$$
W_{n}^{\prime}(t)=\frac{1}{B_{n}} \sum_{j=1}^{[n t]} X_{n j}^{\prime} \text { and } W_{n}^{\prime \prime}(t)=W_{n}(t)-W_{n}^{\prime}(t) .
$$

We shall show first that $W_{n}^{\prime \prime}(t)$ is negligible for the weak convergence on $D[0,1]$ and then, in the next steps, that $W_{n}^{\prime}(t)$ is weakly convergent to the fractional Brownian motion.

In order to explain this step, it is convenient to express the process in an expanded form. By using notation (11)

$W_{n}^{\prime \prime}(t)=\frac{1}{B_{n}} \sum_{i=0}^{[n t]-1} b_{[n t]-i} \varepsilon_{i} I\left(\left|\varepsilon_{i}\right|>\eta_{n-i}\right)+\frac{1}{B_{n}} \sum_{i \geq 1}\left(b_{[n t]+i}-b_{i}\right) \varepsilon_{-i} I\left(\left|\varepsilon_{-i}\right|>\eta_{n+i}\right)$.

We notice that by the triangle inequality,

$$
\begin{gathered}
E\left(\sup _{0 \leq t \leq 1}\left|W_{n}^{\prime \prime}(t)\right|\right) \leq \frac{1}{B_{n}} E\left(\sup _{0 \leq t \leq 1}\left|\sum_{i=0}^{[n t]-1} b_{[n t]-i} \varepsilon_{i} I\left(\left|\varepsilon_{i}\right|>\eta_{n-i}\right)\right|\right) \\
+\frac{1}{B_{n}} E\left(\sup _{0 \leq t \leq 1}\left|\sum_{i \geq 1}\left(b_{[n t]+i}-b_{i}\right) \varepsilon_{-i} I\left(\left|\varepsilon_{-i}\right|>\eta_{n+i}\right)\right|\right) .
\end{gathered}
$$

Then, by monotonicity and using the notation (12)

$$
\begin{gathered}
E\left(\sup _{0 \leq t \leq 1}\left|W_{n}^{\prime \prime}(t)\right|\right) \leq \frac{1}{B_{n}} \sum_{i=0}^{n-1}\left|b_{n-i}\right| E|\varepsilon| I\left(|\varepsilon|>\eta_{n-i}\right) \\
+\frac{1}{B_{n}} \sum_{i \geq 1}\left|b_{n+i}-b_{i}\right| E|\varepsilon| I\left(|\varepsilon|>\eta_{n+i}\right)=\frac{1}{B_{n}} \sum_{i \geq 1}\left|b_{n i}\right| E|\varepsilon| I\left(|\varepsilon|>\eta_{i}\right) .
\end{gathered}
$$

which is exactly the quantity shown to converge to 0 in (16). By Theorem 4.1 in Billingsley (1968), it is enough to study the limiting behavior of $W_{n}^{\prime}(t)$.

Step 5. Tightness.

As before, we reduce the problem to studying the same problem for $W_{n}^{\prime}(t)-$ $E W_{n}^{\prime}(t)$. This is easy to see, since, by the fact the variables are centered and by (20) we clearly obtain

$$
\sup _{0 \leq t \leq 1}\left|E W_{n}^{\prime}(t)\right|=\sup _{0 \leq t \leq 1}\left|E W_{n}^{\prime \prime}(t)\right| \leq E\left(\sup _{0 \leq t \leq 1}\left|W_{n}^{\prime \prime}(t)\right|\right) \rightarrow 0 .
$$

In order to show that $W_{n}^{\prime}(t)-E W_{n}^{\prime}(t)$ is tight in $D[0,1]$ we shall verify the conditions from Lemma 5.5. in Appendix, for the triangular array $B_{n}^{-1}\left(X_{n k}^{\prime}-\right.$ $\left.E X_{n k}^{\prime}\right), 1 \leq k \leq n$. This will be achieved in the following two lemmas. 
By the properties of slowly varying functions (see Seneta 1976 and also Lemma 5.1 in Appendix) we construct first an integer $N_{0}$ and positive constants $K_{i}$ such that for all $m>N_{0}$ we have simultaneously

$$
\begin{gathered}
\max _{1 \leq j \leq m} b_{j}^{2} \leq K_{1} m^{2-2 \alpha} L^{2}(m), \\
l_{2 m} \leq K_{2} l_{m}, \\
\sup _{k>2 m} \frac{\left(b_{k}-b_{k-m}\right)^{2}}{k^{-2 \alpha} L^{2}(k)} \leq K_{3} m^{2}, \\
\sum_{j \geq m} j^{-2 \alpha} L^{2}(j) l_{j} \leq K_{4} m^{1-2 \alpha} L^{2}(m) l_{m} .
\end{gathered}
$$

and

$$
\sum_{j \geq m} j^{-2 \alpha} L^{2}(j) \leq K_{4} m^{1-2 \alpha} L^{2}(m) .
$$

This is possible by Lemma 5.4 and Lemma 5.1 .

Lemma 4.1 There is a constant $K$ and an integers $N_{0}$ such that for any two integers $p$ and $q$ with $1 \leq p<q \leq n$ with $q-p \geq N_{0}$ and any $n \geq N_{0}$

$$
\frac{1}{B_{n}^{2}} \operatorname{var}\left(\sum_{i=p+1}^{q} X_{n i}^{\prime}\right) \leq K\left(\frac{q}{n}-\frac{p}{n}\right)^{2-\alpha} .
$$

Proof. We shall use $N_{0}$ that was already constructed above. We start from the decomposition

$$
\begin{gathered}
\sum_{i=p+1}^{q} X_{n i}^{\prime}=\sum_{i=p}^{q-1} b_{q-i} \varepsilon_{i} I\left(\left|\varepsilon_{i}\right| \leq \eta_{n-i}\right)+\sum_{i=2 p-q}^{p-1}\left(b_{q-i}-b_{p-i}\right) \varepsilon_{i} I\left(\left|\varepsilon_{i}\right| \leq \eta_{n-i}\right) \\
+\sum_{i \geq q-2 p+1}\left(b_{q+i}-b_{p+i}\right) \varepsilon_{-i} I\left(\left|\varepsilon_{-i}\right| \leq \eta_{n+i}\right)=I+I I+I I I
\end{gathered}
$$

We shall estimate the variance of each term separately.

Using the fact that $l_{n}$ is increasing and (22) we obtain

$$
\begin{aligned}
\operatorname{var}(I) & \leq \sum_{i=p}^{q-1} b_{q-i}^{2} l_{n-i}=\sum_{j=1}^{q-p} b_{j}^{2} l_{n-q+j} \leq l_{n}(q-p) \max _{1 \leq j \leq q-p} b_{j}^{2} \\
& \leq K_{1}(q-p)^{3-2 \alpha} L^{2}(q-p) l_{n} .
\end{aligned}
$$

Then, by taking into account that $l_{n}$ is increasing, (22) and (23) we have

$$
\begin{gathered}
\operatorname{var}(I I) \leq \sum_{i=2 p-q}^{p-1}\left(b_{q-i}-b_{p-i}\right)^{2} l_{n-i} \leq l_{2 n} 2(q-p) \max _{1 \leq j \leq 2(q-p)} b_{i}^{2} \\
\leq K_{1} K_{2}(q-p)^{3-2 \alpha} L^{2}(q-p) l_{n} .
\end{gathered}
$$


To estimate the variance of the last term, we use first (24) to obtain

$$
\begin{gathered}
\operatorname{var}(I I I)=\sum_{i \geq q-2 p+1}\left(b_{i+q}-b_{i+p}\right)^{2} l_{n+i} \leq \sum_{j \geq 2(q-p)+1}\left(b_{j}-b_{j-(q-p)}\right)^{2} l_{n+j-q} \\
\leq K_{3}(q-p)^{2} \sum_{j \geq 2(q-p)+1} j^{-2 \alpha} L^{2}(j) l_{n+j-q} .
\end{gathered}
$$

Now, by the monotonicity of $l_{n}$, because $l_{n+j-q} \leq l_{2 n}$ for $j \leq n$ and $l_{n+j-q} \leq l_{2 j}$ for $j>n$ by (23), (25) and (26)

$$
\begin{aligned}
\sum_{j \geq 2(q-p)+1} j^{-2 \alpha} L^{2}(j) l_{n+j-q} & \leq K_{2} K_{5}(q-p)^{-2 \alpha+1} L^{2}(q-p) l_{n} \\
& +K_{2} K_{4}(q-p)^{-2 \alpha+1} L^{2}(q-p) l_{q-p}
\end{aligned}
$$

So, for $K_{6}=K_{2} K_{3}\left(K_{4}+K_{5}\right)$

$$
\operatorname{var}(I I I) \leq K_{6}(q-p)^{3-2 \alpha} L^{2}(q-p) l_{n} .
$$

Overall we have so far for a certain constant $K_{7}$ that does not depend on $p$ or $q$,

$$
\operatorname{var}\left(\sum_{i=p+1}^{q} X_{n i}^{\prime}\right) \leq K_{7}(q-p)^{3-2 \alpha} L^{2}(q-p) l_{n} .
$$

By simple algebra, because $1 \leq p<q \leq n$ we derive

$$
\operatorname{var}\left(\sum_{i=p+1}^{q} X_{n i}^{\prime}\right) \leq K_{7}(q-p)^{2-\alpha} l_{n} n^{1-\alpha} L^{2}(n) \max _{1 \leq k \leq n} \frac{k^{1-\alpha}}{n^{1-\alpha}} \frac{L^{2}(k)}{L^{2}(n)} .
$$

Finally, by the point 5 of Lemma 5.1 ,

$$
\operatorname{var}\left(\sum_{i=p+1}^{q} X_{n i}^{\prime}\right) \leq K_{8}\left(\frac{q}{n}-\frac{p}{n}\right)^{2-\alpha} l_{n} n^{3-2 \alpha} L^{2}(n) .
$$

Therefore, (27) is established by taking into account (7). $\diamond$

Lemma 4.2 Condition (48) is satisfied, namely:

$$
\lim _{n \rightarrow \infty} P\left(\max _{1 \leq k \leq n}\left|X_{n k}^{\prime}-E X_{n k}^{\prime}\right| \geq \varepsilon B_{n}\right)=0 .
$$

Proof. We start from

$$
P\left(\max _{1<k \leq n}\left|X_{n k}^{\prime}-E X_{n k}^{\prime}\right| \geq \varepsilon B_{n}\right) \leq \frac{1}{\varepsilon^{4} B_{n}^{4}} \sum_{k=1}^{n} E\left|X_{n k}^{\prime}-E X_{n k}^{\prime}\right|^{4} .
$$

We use now Rosenthal inequality (Theorem 1.5.13 in de la Peña and Giné 1999), which can be easily extended to an infinite sum of independent random variables, 
by truncating the sum and passing to the limit. So, there is a constant $C$, such that

$E\left|X_{n k}^{\prime}-E X_{n k}^{\prime}\right|^{4} \leq C \sum_{i=1}^{\infty} a_{i}^{4} E \varepsilon^{4} I\left(|\varepsilon| \leq \eta_{n-k+i}\right)+C\left(\sum_{i=1}^{\infty} a_{i}^{2} l_{n-k+i}\right)^{2}=I_{k}+I I_{k}$

By the point 4 of Lemma 5.2 and (15) it follows that

$$
\begin{aligned}
a_{i}^{4} E \varepsilon^{4} I(|\varepsilon| & \left.\leq \eta_{n-k+i}\right) \ll a_{i}^{4}\left(\eta_{n-k+i}^{2}\right) l_{n-k+i} \\
& \ll i^{-4 \alpha} L^{4}(i)(n-k+i) l_{n-k+i}^{2} .
\end{aligned}
$$

So

$$
\sum_{k=1}^{n} I_{k} \leq \sum_{i=1}^{\infty} i^{-4 \alpha} L^{4}(i) \sum_{k=i}^{n+i} k l_{k}^{2} \ll n^{2} l_{n}^{2}
$$

Then, by simple computations involving the partition of sum in two parts, one up to $2 n$ and the rest, and then using the properties of regularly functions and the fact that $2 \alpha>1$ we obtain

$$
\sum_{k=1}^{n} I I_{k} \leq n\left(\sum_{i=1}^{\infty} i^{-2 \alpha} L^{2}(i) l_{n+i}\right)^{2} \leq n l_{n}^{2}
$$

Finally by (7) we notice that

$$
\frac{n^{2} l_{n}^{2}}{B_{n}^{4}} \rightarrow 0
$$

$\diamond$

Step 6. Convergence of finite dimensional distributions.

Let $0 \leq t_{1}<t_{2}<\cdots<t_{m} \leq 1$. We shall show next that the vector $\left(W_{n}^{\prime}\left(t_{j}\right) ; 1 \leq j \leq m\right)$ converges in distribution to the finite dimensional distributions of a fractional Brownian motion with Hurst index $3 / 2-2 \alpha$, i.e. of a Gaussian process with covariance structure $\frac{1}{2}\left(t^{3-2 \alpha}+s^{3-2 \alpha}-(t-s)^{3-2 \alpha}\right)$ for $s<t$.

By the Cramér-Wold device and taking into account (21) we have to study the limiting distribution of $\sum_{j=2}^{m} \lambda_{j}\left(W_{n}^{\prime}\left(t_{j}\right)-E W_{n}^{\prime}\left(t_{j-1}\right)\right)$, which we express as a weighted sum of independent random variables. By elementary computations involving similar arguments used in the proof of step 3, and taking into account (16) and (19), we notice that Lyapunov's condition is satisfied and then, the limiting distribution is normal with the covariance structure that will be specified next. We compute now the covariance of $W_{n}^{\prime}(s)$ and $W_{n}^{\prime}(t)$ for $s \leq t$. By simple algebra

$$
\operatorname{cov}\left(W_{n}^{\prime}(t), W_{n}^{\prime}(s)\right)=\frac{1}{2}\left(\operatorname{var}\left(W_{n}^{\prime}(t)\right)+\operatorname{var}\left(W_{n}^{\prime}(s)\right)-\operatorname{var}\left(W_{n}^{\prime}(t)-W_{n}^{\prime}(s)\right)\right) .
$$


We analyze now the variance of $W_{n}^{\prime}(t)$. For each $t$ fixed, $0 \leq t \leq 1$

$$
\begin{gathered}
\operatorname{var}\left(W_{n}^{\prime}(t)\right)=\frac{1}{B_{n}^{2}} \sum_{i=0}^{[n t]-1} b_{[n t]-i}^{2}\left(E \varepsilon_{0}^{2} I\left(\left|\varepsilon_{0}\right| \leq \eta_{n-i}\right)-E^{2} \varepsilon_{0} I\left(\left|\varepsilon_{0}\right| \leq \eta_{n-i}\right)\right) \\
+\frac{1}{B_{n}^{2}} \sum_{i \geq 1}\left(b_{[n t]+i}-b_{i}\right)^{2}\left(E \varepsilon_{0}^{2} I\left(\left|\varepsilon_{0}\right| \leq \eta_{n+i}\right)-E^{2} \varepsilon_{0} I\left(\left|\varepsilon_{0}\right| \leq \eta_{n+i}\right)\right) .
\end{gathered}
$$

Taking into account $E \varepsilon_{0} I\left(\left|\varepsilon_{0}\right| \leq \eta_{n-i}\right)=-E \varepsilon_{0} I\left(\left|\varepsilon_{0}\right|>\eta_{n-i}\right)$, by Lemma 5.2 and Lemma 5.4, after some computations, we obtain

$$
\operatorname{var}\left(W_{n}^{\prime}(t)\right) \sim \frac{1}{B_{n}^{2}} \sum_{i=0}^{[n t]-1} b_{[n t]-i}^{2} l_{n-i}+\frac{1}{B_{n}^{2}} \sum_{i \geq 1}\left(b_{[n t]+i}-b_{i}\right)^{2} l_{n+i} .
$$

With a similar proof as of relation (38) of Lemma 5.4, for every $0 \leq t \leq 1$

$$
\operatorname{var}\left(W_{n}^{\prime}(t)\right) \rightarrow t^{3-2 \alpha}
$$

and for every $0 \leq s<t \leq 1$

$$
\operatorname{var}\left(W_{n}^{\prime}(t)-W_{n}^{\prime}(s)\right) \rightarrow(t-s)^{3-2 \alpha} .
$$

Then

$$
\operatorname{cov}\left(W_{n}^{\prime}(t), W_{n}^{\prime}(s)\right) \rightarrow \frac{1}{2}\left(t^{3-2 \alpha}+s^{3-2 \alpha}-(t-s)^{3-2 \alpha}\right),
$$

that is the desired covariance structure. $\diamond$

\subsection{Proof of Theorem 2.2}

We notice that it is enough to prove only the convergence in (9). Then (4), (7) and (9) imply

$$
B_{n}^{2} \sim c_{\alpha} n^{2} a_{n}^{2}\left(\sum_{j=1}^{n} X_{j}^{2}\right) / A^{2}
$$

which we combine with Theorem 2.1, via Slutsky's theorem, to obtain the selfnormalized part of the theorem. The proof of (9) will be decomposed in several steps.

Step 1. Truncation.

Denote $D_{n}^{2}=A^{2} n l_{n}$. Recall the definition (10) and set $X_{n j}^{\prime \prime}=X_{j}-X_{n j}^{\prime}$. In order to prove (9) it is enough to establish

$$
F_{n}=\sum_{j=1}^{n}\left(X_{n j}^{\prime \prime}\right)^{2} / D_{n}^{2} \stackrel{P}{\rightarrow} 0
$$

and

$$
G_{n}=\sum_{j=1}^{n}\left(X_{n j}^{\prime}\right)^{2} / D_{n}^{2} \stackrel{P}{\rightarrow} 1
$$


To see this we square the decomposition $X_{j}=X_{n j}^{\prime}+X_{n j}^{\prime \prime}$; then sum with $j$ from 1 to $n$, and notice that by the Hölder inequality

$$
F_{n}-2\left(G_{n} F_{n}\right)^{1 / 2} \leq \frac{1}{D_{n}^{2}} \sum_{j=1}^{n} X_{j}^{2}-G_{n} \leq F_{n}+2\left(G_{n} F_{n}\right)^{1 / 2} .
$$

Step 2. Proof of (30).

We start from

$$
\begin{gathered}
\sum_{k=1}^{n}\left(X_{n k}^{\prime \prime}\right)^{2}=\sum_{k=1}^{n} \sum_{i=1}^{\infty} a_{i}^{2} \varepsilon_{k-i}^{2} I\left(\left|\varepsilon_{k-i}\right|>\eta_{n-k+i}\right)+ \\
2 \sum_{k=1}^{n} \sum_{i<j} a_{i} a_{j} \varepsilon_{k-i} I\left(\left|\varepsilon_{k-i}\right|>\eta_{n-k+i}\right) \varepsilon_{k-j} I\left(\left|\varepsilon_{k-j}\right|>\eta_{n-k+j}\right)=I+I I .
\end{gathered}
$$

(here and below $\sum_{i<j}$ denotes double summation). By independence, monotonicity, and the point 3 of Lemma 5.2. we easily deduce that

$$
\begin{gathered}
\quad E|I I| \leq 2 \sum_{k=1}^{n} \sum_{i<j}\left|a_{i} a_{j}\right| E\left|\varepsilon_{k-i} I\left(\left|\varepsilon_{k-i}\right|>\eta_{n-k+i}\right) \varepsilon_{k-j} I\left(\left|\varepsilon_{k-j}\right|>\eta_{n-k+j}\right)\right| \\
\leq 2 n \sum_{i<j}\left|a_{i} a_{j}\right| E|\varepsilon| I\left(|\varepsilon|>\eta_{i}\right) E|\varepsilon| I\left(|\varepsilon|>\eta_{j}\right)=2 n \sum_{i<j}\left|a_{i} a_{j}\right| o\left(\eta_{i}^{-1} l_{i}\right) o\left(\eta_{j}^{-1} l_{j}\right) .
\end{gathered}
$$

Then, by (15), clearly

$$
E|I I| \leq n\left(\sum_{i \geq 1}\left|a_{i}\right| i^{-1 / 2} o\left(l_{i}^{1 / 2}\right)\right)^{2} .
$$

Because $\sum_{i \geq 1}\left|a_{i}\right| i^{-1 / 2}<\infty$, and $l_{n}$ is increasing, it is easy to see that

$$
E|I I|=o\left(n l_{n}\right)=o\left(D_{n}^{2}\right) .
$$

In order to estimate the contribution of the term $I$, by changing the order of summation we express this term in the following way

$$
I=\sum_{j=1}^{n}\left(\sum_{i=1}^{j} a_{i}^{2}\right) \varepsilon_{n-j}^{2} I\left(\left|\varepsilon_{n-j}\right|>\eta_{j}\right)+\sum_{j=n+1}^{\infty}\left(\sum_{i=j-n+1}^{j} a_{i}^{2}\right) \varepsilon_{n-j}^{2} I\left(\left|\varepsilon_{n-j}\right|>\eta_{j}\right) .
$$

We implement now the notation

$$
A_{n j}^{2}=A_{j}^{2}=\sum_{i=1}^{j} a_{i}^{2} \text { when } j \leq n \text { and } A_{n j}^{2}=\sum_{i=j-n+1}^{j} a_{i}^{2} \text { when } j>n .
$$

and then we express $I$ as

$$
I=\sum_{j=1}^{\infty} A_{n j}^{2} \varepsilon_{n-j}^{2} I\left(\left|\varepsilon_{n-j}\right|>\eta_{i}\right) .
$$


Clearly $A_{n j}^{2}$ are uniformly bounded by a constant. In addition, by relation (41), for $j>2 n$, these coefficients have the following order of magnitude

$$
\begin{aligned}
A_{n j}^{2} & \ll n^{2}(j-n)^{-2 \alpha-1} \max _{j-n \leq k \leq j} L^{2}(k) \leq n^{2}(j-n)^{-2 \alpha-1} \max _{j / 2 \leq k \leq j} L^{2}(k) \\
& \ll n^{2}(j-n)^{-2 \alpha-1} \min _{j / 2 \leq k \leq j} L^{2}(k) \leq n^{2}(j-n)^{-2 \alpha-1} L^{2}(j) .
\end{aligned}
$$

Now, we use first the Khinchin's inequality (see Lemma 1.4.13 in de la Peña and Giné, 1999) followed by the triangle inequality and Lemma 5.2, and relation (15) to obtain

$$
E \sqrt{I} \ll E\left|\sum_{j=1}^{\infty} A_{n j} \varepsilon_{n-j} I\left(\left|\varepsilon_{n-j}\right|>\eta_{j}\right)\right|=\sum_{j=1}^{\infty} A_{n j} o\left(\eta_{j}^{-1} l_{j}\right)=\sum_{j=1}^{\infty} A_{n j} j^{-1 / 2} o\left(l_{j}^{1 / 2}\right) .
$$

We notice that by (33), the point 4 of Lemma 5.1 and the fact that $\alpha>1 / 2$,

$$
\begin{aligned}
E \sqrt{I} & =o\left(\sqrt{n l_{n}}\right)+n \sum_{j \geq n}^{\infty} j^{-\alpha-1 / 2} L^{2}(j+n) j^{-1 / 2} o\left(l_{j}^{1 / 2}\right) \\
& =o\left(\sqrt{n l_{n}}\right)+O\left(n^{-\alpha} L^{2}(n) l_{j}^{1 / 2}\right)=o\left(\sqrt{n l_{n}}\right) .
\end{aligned}
$$

As a consequence, $\sqrt{I / n l_{n}}$ converges in $L_{1}$ to 0 , and so, $I / D_{n}^{2}$ is convergent to 0 in probability. By gathering all these facts we deduce that (30) holds and the proof is reduced to show that (31) holds.

Step 3. Proof of (31).

We express the sum of squares as

$$
\begin{gathered}
\sum_{k=1}^{n}\left(X_{k}^{\prime}\right)^{2}=\sum_{k=1}^{n} \sum_{i=1}^{\infty} a_{i}^{2} \varepsilon_{k-i}^{2} I\left(\left|\varepsilon_{k-i}\right| \leq \eta_{n-k+i}\right) \\
+2 \sum_{k=1}^{n} \sum_{1 \leq i<j} a_{i} a_{j} \varepsilon_{k-i} I\left(\left|\varepsilon_{k-i}\right| \leq \eta_{n-k+i}\right) \varepsilon_{k-j} I\left(\left|\varepsilon_{k-j}\right| \leq \eta_{n-k+j}\right) .
\end{gathered}
$$

We shall show that

$$
\frac{1}{D_{n}^{2}} \sum_{k=1}^{n} \sum_{i=1}^{\infty} a_{i}^{2} \varepsilon_{k-i}^{2} I\left(\left|\varepsilon_{k-i}\right| \leq \eta_{n-k+i}\right) \stackrel{P}{\rightarrow} 1
$$

and

$$
\frac{1}{D_{n}^{2}} \sum_{k=1}^{n} \sum_{1 \leq i<j} a_{i} a_{j} \varepsilon_{k-i} I\left(\left|\varepsilon_{k-i}\right| \leq \eta_{n-k+i}\right) \varepsilon_{k-j} I\left(\left|\varepsilon_{k-j}\right| \leq \eta_{n-k+j}\right) \stackrel{P}{\rightarrow} 0 .
$$

We establish first (34).

By using the notation (32), we have

$$
\sum_{k=1}^{n} \sum_{i=1}^{\infty} a_{i}^{2} \varepsilon_{k-i}^{2} I\left(\left|\varepsilon_{k-i}\right| \leq \eta_{n-k+i}\right)=\sum_{i=1}^{\infty} A_{n i}^{2} \varepsilon_{n-i}^{2} I\left(\left|\varepsilon_{n-i}\right| \leq \eta_{i}\right) .
$$


By independence, part 4 of Lemma [5.2, relations (33) and (15), and taking into account that $\alpha>1 / 2$ we get

$$
\begin{aligned}
& \operatorname{Var}\left(\sum_{i=1}^{\infty} A_{n i}^{2} \varepsilon_{n-i}^{2} I\left(\left|\varepsilon_{n-i}\right| \leq \eta_{i}\right)\right) \leq \sum_{i=1}^{\infty} A_{n i}^{4} E \varepsilon^{4} \mathbf{1}\left(|\varepsilon| \leq \eta_{i}\right) \\
& =\sum_{i=1}^{2 n} \eta_{i}^{2} o\left(l_{i}\right)+\sum_{i \geq 2 n}(i-n)^{-4 \alpha-2} L^{4}(i) n^{4} \eta_{i}^{2} o\left(l_{i}\right)=o\left(n^{2} l_{n}^{2}\right)=o\left(D_{n}^{4}\right) .
\end{aligned}
$$

So (34) is reduced to showing that

$$
\frac{1}{D_{n}^{2}} \sum_{i=1}^{\infty} A_{n i}^{2} E\left(\varepsilon^{2} I\left(|\varepsilon| \leq \eta_{i}\right)=\frac{1}{D_{n}^{2}} \sum_{i=1}^{\infty} A_{n i}^{2} l_{i} \rightarrow 1 \text { as } n \rightarrow \infty .\right.
$$

We divide the sum in three parts, one from 1 to $n$, one from $n+1$ to $2 n$ and the rest of the series. We easily see that by (33),

$$
\sum_{j=2 n+1}^{\infty} A_{n j}^{2} l_{j} \ll \sum_{j=2 n+1}^{\infty}(j-n)^{-2 \alpha-1} n^{2} L^{2}(j) l_{j}=o\left(D_{n}^{2}\right) .
$$

Then,

$$
\begin{gathered}
\sum_{j=n+1}^{2 n} A_{n j}^{2} l_{j} \ll \sum_{j=n+1}^{2 n}(j-n)^{1-2 \alpha} l_{j} \max _{1 \leq i \leq n} L(i) \\
\ll\left(\sum_{k=1}^{n} k^{1-2 \alpha} l_{n+k}\right) \max _{1 \leq i \leq n} L(i)=o\left(D_{n}^{2}\right) .
\end{gathered}
$$

Now, by the proof of relation (42) in Appendix with the only difference that we replace $a_{i}$ by $a_{i}^{2}$ and so $b_{n}^{2}$ by $A_{j}^{2}$ we obtain

$$
\sum_{j=1}^{n} A_{j}^{2} l_{j} \sim l_{n} \sum_{j=1}^{n} A_{j}^{2}
$$

Finally, by (36), the definition of $D_{n}^{2}$ and by the Toeplitz lemma, (44) in Appendix, it follows

$$
\lim _{n \rightarrow \infty} \frac{1}{D_{n}^{2}} \sum_{j=1}^{n} A_{j}^{2} l_{j}=\lim _{n \rightarrow \infty} \frac{l_{n} \sum_{j=1}^{n} A_{j}^{2}}{l_{n} n A^{2}}=\lim _{n \rightarrow \infty} \frac{A_{n}^{2}}{A^{2}}=1 .
$$

This completes the proof of (34).

We move now to prove (35). Let $N$ be a fixed positive integer. For each $1 \leq k \leq n$ we divide the sum in two parts:

$$
\begin{aligned}
& \sum_{j=1}^{N} \sum_{i=1}^{j-1} a_{i} a_{j} \varepsilon_{k-i} I\left(\left|\varepsilon_{k-i}\right| \leq \eta_{n-k+i}\right) \varepsilon_{k-j} I\left(\left|\varepsilon_{k-j}\right| \leq \eta_{n-k+j}\right) \\
+ & \sum_{j>N} \sum_{i=1}^{j-1} a_{i} a_{j} \varepsilon_{k-i} I\left(\left|\varepsilon_{k-i}\right| \leq \eta_{n-k+i}\right) \varepsilon_{k-j} I\left(\left|\varepsilon_{k-j}\right| \leq \eta_{n-k+j}\right)=I_{k}+I I_{k} .
\end{aligned}
$$


We estimate the variance of the sum of each term separately.

For estimating $\operatorname{var}\left(\sum_{k=1}^{n} I I_{k}\right)$ we apply the Hölder inequality:

$\operatorname{var}\left(\sum_{k=1}^{n} I I_{k}\right) \leq n \sum_{k=1}^{n} \operatorname{var}\left(\sum_{j>N} \sum_{i=1}^{j-1} a_{i} a_{j} \varepsilon_{k-i} I\left(\left|\varepsilon_{k-i}\right| \leq \eta_{n-k+i}\right) \varepsilon_{k-j} I\left(\left|\varepsilon_{k-j}\right| \leq \eta_{n-k+j}\right)\right)$.

By independence, a term corresponding to the combination of indexes $\left(k-i_{1}, k-\right.$ $\left.j_{1}, k-i_{2}, k-j_{2}\right)$ with $i_{1}<j_{1}$ has a non-null contribution if and only if $i_{1}=i_{2}$ and $j_{1}=j_{2}$, leading to

$$
\operatorname{var}\left(\sum_{k=1}^{n} I I_{k}\right) \leq n^{2} \sum_{j>N} \sum_{i=1}^{j-1} a_{i}^{2} a_{j}^{2} l_{n+i} l_{n+j}=\left(n^{2} l_{n}^{2}\right) o_{N}(1),
$$

where we used first the monotonicity of $l_{n}$ and in the last part we used the fact that (by monotonicity, the definition of slowly varying functions and our notations) $l_{i} \leq l_{2 n} \ll l_{n}$, for $i \leq 2 n$ and $l_{i+n} \leq l_{3 i / 2} \ll l_{i}$ for $i>2 n$ along with the convergence of the series $\sum_{i} a_{i}^{2} l_{i}$.

In order to treat the other term we start from

$\operatorname{var}\left(\sum_{k=1}^{n} I_{k}\right)=\operatorname{var}\left(\sum_{j=1}^{N} \sum_{i=1}^{j-1} a_{i} a_{j} \sum_{k=1}^{n} \varepsilon_{k-i} I\left(\left|\varepsilon_{k-i}\right| \leq \eta_{n-k+i}\right) \varepsilon_{k-j} I\left(\left|\varepsilon_{k-j}\right| \leq \eta_{n-k+j}\right)\right)$

and then, because we compute the variance of at most $N^{2}$ sums and because the coefficients $a_{i}$ are bounded, clearly,

$\operatorname{var}\left(\sum_{k=1}^{n} I_{k}\right) \ll N^{4} \max _{1 \leq i<j \leq N} \operatorname{var}\left(\sum_{k=1}^{n} \varepsilon_{k-i} I\left(\left|\varepsilon_{k-i}\right| \leq \eta_{n-k+i}\right) \varepsilon_{k-j} I\left(\left|\varepsilon_{k-j}\right| \leq \eta_{n-k+j}\right)\right)$.

We notice now that

$$
\begin{aligned}
& \operatorname{var}\left(\sum_{k=1}^{n} \varepsilon_{k-i} I\left(\left|\varepsilon_{k-i}\right| \leq \eta_{n-k+i}\right) \varepsilon_{k-j} I\left(\left|\varepsilon_{k-j}\right| \leq \eta_{n-k+j}\right)\right) \\
\leq & \left.\sum_{k=1}^{n} E \varepsilon_{k-i}^{2} I\left(\left|\varepsilon_{k-i}\right| \leq \eta_{n-k+i}\right) E \varepsilon_{k-j}^{2} I\left(\left|\varepsilon_{k-j}\right| \leq \eta_{n-k+j}\right)\right),
\end{aligned}
$$

since by independence and the fact that $i \neq j$ all the other terms are equal to 0 . The result is

$$
\operatorname{var}\left(\sum_{k=1}^{n} I_{k}\right) \ll N^{4} \sum_{k=1}^{n} l_{n, n-k+i} l_{n, n-k+j} \ll N^{4}\left(n l_{n}^{2}\right) .
$$

Overall

$$
\begin{gathered}
\frac{1}{D_{n}^{4}} \operatorname{var}\left(\sum_{k=1}^{n} \sum_{1 \leq i<j} \varepsilon_{k-i} I\left(\left|\varepsilon_{k-i}\right| \leq \eta_{n-k+i}\right) \varepsilon_{k-j} I\left(\left|\varepsilon_{k-j}\right| \leq \eta_{n-k+j}\right)\right) \leq \\
\frac{2}{D_{n}^{4}} \operatorname{var}\left(\sum_{k=1}^{n} I_{k}\right)+\frac{2}{D_{n}^{4}} \operatorname{var}\left(\sum_{k=1}^{n} I I_{k}\right)=o_{N}(1)+O\left(N^{4} \frac{1}{n}\right) .
\end{gathered}
$$

We conclude that (35) holds by letting first $n \rightarrow \infty$ followed by $N \rightarrow \infty$. $\diamond$ 


\section{Appendix}

We formulate in the first lemma several properties of the slowly varying function. Their proofs can be found in Seneta (1976).

Lemma 5.1 A slowly varying function $l(x)$ defined on $[A, \infty)$ has the following properties:

1. There exists $B \geq A$ such that for all $x \geq B, l(x)$ is representable in the form $l(x)=g(x) \exp \left(\int_{B}^{x} \frac{a(y)}{y} d y\right)$, where $g(x) \rightarrow c_{0}>0$, and $a(x) \rightarrow 0$ as $x \rightarrow \infty$. In addition $a(x)$ is continuous.

2. For $B<c<C<\infty, \lim _{x \rightarrow \infty} \frac{l(t x)}{l(x)}=1$ uniformly in $c \leq t \leq C$.

3. For any $\theta>-1, \int_{B}^{x} y^{\theta} l(y) d y \sim \frac{x^{\theta+1} l(x)}{\theta+1}$ as $x \rightarrow \infty$.

4. For any $\theta<-1, \int_{x}^{\infty} y^{\theta} l(y) d y \sim \frac{x^{\theta+1} l(x)}{-\theta-1}$ as $x \rightarrow \infty$.

5. For any $\eta>0, \sup _{t \geq x}\left(t^{\eta} l(t)\right) \sim x^{\eta} l(x)$ as $x \rightarrow \infty$. Moreover $\sup _{t \geq x}\left(t^{\eta} l(t)\right)=$ $x^{\eta} \bar{l}(x)$ where $\bar{l}(x)$ is slowly varying and $\bar{l}(x) \sim l(x)$.

The following lemma contains some equivalent formulation for variables in the domains of attraction of normal law (3). It is Lemma 1 in Csörgő et al (2003); see also Feller (1966).

Lemma 5.2 The following statements are equivalent:

1. $l(x)=E X^{2} I(|X| \leq x)$ is a slowly varying function at $\infty$;

2. $P(|X|>x)=o\left(x^{-2} l(x)\right)$;

3. $E|X| I(|X|>x)=o\left(x^{-1} l(x)\right)$;

4. $E|X|^{\alpha} I(|X| \leq x)=o\left(x^{\alpha-2} l(x)\right)$ for $\alpha>2$.

To clarify the behavior of the sequence of normalizer $B_{n}^{2}$ defined by (7) we state the following lemma that follows from relations (3.33) and (3.44) in Kuelbs (1985).

Lemma 5.3 Assume (3) and define $\eta_{n}$ by (6). Then, $l_{n}=l\left(\eta_{n}\right)$ is a slowly varying function at $\infty$.

The next lemma is useful to study the variance of partial sums for truncated random variables.

Lemma 5.4 Under conditions of Theorem 2.1 and with the notation (8) and (12) we have 
1. The coefficients have the following order of magnitude: There are constants $C_{1}$ and $C_{2}$ such that for all $n \geq 1$,

$$
\begin{gathered}
\left|b_{n i}\right| \leq C_{1} i^{1-\alpha}|L(i)| \text { for } i \leq 2 n \text { and }\left|b_{n i}\right| \leq C_{2} n(i-n)^{-\alpha}|L(i)| \text { for } i>2 n \\
\qquad \sum_{i=1}^{\infty} b_{n i}^{2} \sim c_{\alpha} n^{3-2 \alpha} L^{2}(n)
\end{gathered}
$$

2. The asymptotic equivalence for the variance:

$$
\sum_{i \geq 1} b_{n i}^{2} l_{i} \sim l_{n} \sum_{i \geq 1} b_{n i}^{2} \sim B_{n}^{2}
$$

where $B_{n}^{2}$ is defined by (7).

3. For any $p \geq 1$ and any function $h(x)$ slowly varying at $\infty$,

$$
\sum_{i \geq 1}\left|b_{n i}\right|^{p} i^{-1+p / 2}|h(i)| \ll h(n) n^{p(3 / 2-\alpha)} L^{p}(n) .
$$

Proof. The fact that $\left|b_{n i}\right| \leq C_{1} i^{1-\alpha}|L(i)|$ for $i \leq 2 n$ follows easily by the properties of slowly varying functions listed in Lemma 5.1

For $i>2 n$, by the properties of strong slowly varying functions, for $n$ sufficiently large:

$$
(i-n)^{-\alpha} L(i-n)+\ldots+i^{-\alpha} L(i) \leq\left[(i-n)^{-\alpha}+\ldots+i^{-\alpha}\right] \max _{i-n \leq j \leq i} L(j) .
$$

Then,

$$
\max _{i-n \leq j \leq i} L(j) \leq \max _{i / 2 \leq j \leq i} L(j) \ll L(i)
$$

since

$$
\frac{\max _{m \leq j \leq 2 m} L(j)}{\min _{m \leq j<2 m} L(j)} \rightarrow 1
$$

The asymptotic equivalence in (38) is well known. See for instance Theorem 2 in $\mathrm{Wu}$ and Min (2005).

We turn now to show (39). Let $M$ be a positive integer. We divide the sum in 3 parts, one from 1 to $n$, one from $n+1$ to $n M$, and the third one with all the other terms. The idea of the proof is that for $n$ and $M$ large, the sum from 1 to $n M$ dominates the sum of the rest of the terms.

We treat each of these three sums separately.

By using the definition of $b_{n i}=a_{1}+\ldots+a_{i}=b_{i}$ for $1 \leq i \leq n$ by analogy with the point 3 in Lemma 5.1 we show that

$$
\sum_{i=1}^{n} b_{i}^{2} l_{i} \sim l_{n} \sum_{i=1}^{n} b_{i}^{2}
$$


To see this, by the first part of Lemma (5.1) we have $l_{n}=g_{n} h_{n}$ where $h_{n}=$ $\exp \left(\int_{B}^{n} \frac{a(y)}{y} d y\right), g_{n} \rightarrow c>0, a(x) \rightarrow 0$ as $x \rightarrow \infty$, and $a(x)$ is continuous. It is easy to show that

$$
h_{n}-h_{n-1}=o\left(h_{n} / n\right) \text { as } n \rightarrow \infty
$$

and also, by the part 3 of the same lemma, we get $\sum_{i=1}^{n-1} b_{i}^{2} \ll n b_{n}^{2}$.

Next, we have just to use the well known Toeplitz lemma:

$$
\lim _{n \rightarrow \infty} \frac{c_{n}}{d_{n}}=\lim _{n \rightarrow \infty} \frac{c_{n}-c_{n-1}}{d_{n}-d_{n-1}}
$$

provided $d_{n} \rightarrow \infty$ and the limit in the right hand side exists. Then, it follows that

$$
\lim _{n \rightarrow \infty} \frac{\sum_{i=1}^{n} b_{i}^{2} l_{i}}{l_{n} \sum_{i=1}^{n} b_{i}^{2}}=\lim _{n \rightarrow \infty} \frac{\sum_{i=1}^{n} b_{i}^{2} l_{i}}{c h_{n} \sum_{i=1}^{n} b_{i}^{2}}=\lim _{n \rightarrow \infty} \frac{b_{n}^{2} h_{n}}{h_{n} \sum_{i=1}^{n} b_{i}^{2}-h_{n-1} \sum_{i=1}^{n-1} b_{i}^{2}} .
$$

We shall show that the limit in the right hand side is equal to 1 . We start by writing

$$
h_{n} \sum_{i=1}^{n} b_{i}^{2}-h_{n-1} \sum_{i=1}^{n-1} b_{i}^{2}=\left(h_{n}-h_{n-1}\right) \sum_{i=1}^{n-1} b_{i}^{2}+b_{n}^{2} h_{n} .
$$

Then, by (43)

$$
\lim _{n \rightarrow \infty} \frac{b_{n}^{2} h_{n}}{h_{n} \sum_{i=1}^{n} b_{i}^{2}-h_{n-1} \sum_{i=1}^{n-1} b_{i}^{2}}=\lim _{n \rightarrow \infty} \frac{b_{n}^{2} h_{n}}{o\left(h_{n} / n\right) \sum_{i=1}^{n} b_{i}^{2}+b_{n}^{2} h_{n}}=1,
$$

and (42) follows.

To treat the second sum, notice that $l_{n}$ is increasing and then

$$
\sum_{i=n+1}^{n M} b_{n i}^{2} l_{i} \sim l_{n} \sum_{i=n+1}^{n M} b_{n i}^{2}
$$

because

$$
l_{n} \sum_{i=n+1}^{n M} b_{n i}^{2} \leq \sum_{i=n+1}^{n M} b_{n i}^{2} l_{i} \leq l_{n M} \sum_{i=n+1}^{n M} b_{n i}^{2}
$$

and $l_{n}$ is a function slowly varying at $\infty$.

We treat now the last sum. By (38), and Lemma 5.1

$$
\sum_{i=n M+1}^{\infty} b_{n i}^{2} l_{i} \ll n^{2} \sum_{i=n M+1}^{\infty}(i-n)^{-2 \alpha} L^{2}(i) l_{i} \ll n^{2}[n(M-1)]^{1-2 \alpha} L^{2}[n M] l_{n M} .
$$

We obtain

$$
\sum_{i=n M+1}^{\infty} b_{n i}^{2} l_{i} \ll B_{n}^{2} M^{1-2 \alpha} \text { as } n \rightarrow \infty
$$


We combine now the estimates in (42) and (45). For $\delta>0$ fixed and $n$ sufficiently large

$$
(1-\delta) l_{n} \sum_{i \geq 1}^{n M} b_{n i}^{2} \leq \sum_{i \geq 1}^{n M} b_{n i}^{2} l_{i} \leq(1+\delta) l_{n} \sum_{i \geq 1}^{n M} b_{n i}^{2}
$$

Therefore,

$$
(1-\delta) l_{n}\left(\sum_{i \geq 1}^{\infty} b_{n i}^{2}-\sum_{i>n M} b_{n i}^{2}\right) \leq \sum_{i \geq 1}^{\infty} b_{n i}^{2} l_{i} \leq(1+\delta) l_{n} \sum_{i \geq 1}^{n M} b_{n i}^{2}+\sum_{i>n M} b_{n i}^{2} l_{i} .
$$

Then, by (46), for a positive constant $C_{1}$ we have

$$
\lim \sup _{n \rightarrow \infty} \frac{1}{B_{n}^{2}} \sum_{i>n M} b_{n i}^{2} l_{i} \leq \frac{C_{1}}{M^{2 \alpha-1}} .
$$

We also know that for a certain positive constant $C_{2}$,

$$
\lim \sup _{n \rightarrow \infty} \frac{1}{B_{n}^{2}} \sum_{i>n M} b_{n i}^{2} \leq \frac{C_{2}}{M^{2 \alpha-1}} .
$$

The result follows by dividing (47) by $B_{n}^{2}$ and taking first lim sup and also lim inf when $n \rightarrow \infty$ followed by $M \rightarrow \infty$, and finally we let $\delta \rightarrow 0$.

The proof of (40) is similar and it is sufficient to divide the sum in only two parts, one from 1 to $2 n$ and the rest. More exactly by using (37),

$$
\sum_{i=1}^{2 n}\left|b_{n i}\right|^{p} i^{-1+p / 2}|h(i)| \ll \sum_{i=1}^{2 n} i^{p(1-\alpha)}|L(i)|^{p} i^{-1+p / 2}|h(i)| \ll h(n) n^{p(3 / 2-\alpha)} L^{p}(n)
$$

and,

$\sum_{i \geq 2 n}\left|b_{n i}\right|^{p} i^{-1+p / 2}|h(i)| \ll n^{p} \sum_{i \geq 2 n} i^{-1+(1 / 2-\alpha) p}|L(i)|^{p}|h(i)| \ll h(n) n^{p(3 / 2-\alpha)} L^{p}(n)$.

The proof is complete. $\diamond$

Next lemma is a variant of Theorem 12.3 in Billingsley (1968).

Lemma 5.5 Assume that $\left(X_{n k}\right)_{1 \leq k \leq n}$ is a triangular array of centered random variables with finite second moment. For $0 \leq m \leq n$ let $S_{m}=\sum_{j=1}^{m} X_{n j}$ and for $0 \leq t \leq 1, W_{n}(t)=S_{[n t]}$. Assume that for every $\varepsilon>0$

$$
P\left(\max _{1 \leq i \leq n}\left|X_{n i}\right|>\varepsilon\right) \rightarrow 0
$$

and there is a positive constant $K$, and an integer $N_{0}$ such that for any $1 \leq p<$ $q \leq n$ with $q-p>N_{0}$ we have

$$
E\left(S_{n q}-S_{n p}\right)^{2} \leq K\left(\frac{q}{n}-\frac{p}{n}\right)^{\gamma}
$$

for some $\gamma>1$. Then $W_{n}(t)$ is tight in $D[0,1]$, endowed with Skorohod topology. 
Proof. We shall base our proof on a blocking argument. We divide the variables in blocks of size $N_{0}$. Let $k=\left[n / N_{0}\right]$. For $1 \leq j \leq k$ denote $Y_{n j}=$ $\sum_{i=(j-1) N_{0}+1}^{j N_{0}} X_{n i}$ and $Y_{n, k+1}=\sum_{i=k N_{0}+1}^{n} X_{n i}$. Define $V_{n}(t)=\sum_{j=1}^{[k t]} Y_{n k}$.

Then we notice that it is enough to show that $V_{n}(t)$ is tight in $D[0,1]$ because by the fact that $[n t]-[k t] \leq 2 N_{0}$ and by (48)

$$
P\left(\sup _{t}\left|W_{n}(t)-V_{n}(t)\right|>\varepsilon\right) \leq P\left(\max _{1 \leq i \leq n}\left|X_{n i}\right|>\varepsilon / 2 N_{0}\right) \rightarrow 0 .
$$

By Theorem 8.3 in Billingsley (1968) formulated for random elements of D (see page 137 in Billingsley, 1968) we have to show that for every $0 \leq t \leq 1$ and $\varepsilon>0$ fixed,

$$
\lim _{\delta \searrow 0} \lim \sup _{n \rightarrow \infty} \frac{1}{\delta} P\left(\max _{[k t] \leq j \leq[k(t+\delta)]}\left|\sum_{i=[k t]}^{j} Y_{n i}\right| \geq \varepsilon\right)=0 .
$$

By Theorem 12.2 in Billingsley (1968), because $\gamma>1$, there is a constant $K$ such that

$$
P\left(\max _{[k t] \leq j \leq[k(t+\delta)]}\left|\sum_{i=[k t]}^{j} Y_{n i}\right| \geq \varepsilon\right) \leq \frac{K}{\varepsilon^{2}}\left(\left[\frac{k(t+\delta) N_{0}}{n}\right]-\left[\frac{k t N_{0}}{n}\right]\right)^{\gamma}
$$

and the result follows by multiplying with $1 / \delta$ and passing to the limit with $n \rightarrow \infty$ and then with $\delta \rightarrow 0$. $\diamond$

\section{Acknowledgement}

The authors are grateful to the referees for carefully reading the paper and for numerous suggestions that significantly improved the presentation of the paper.

\section{References}

[1] Billingsley, P. (1968). Convergence of Probability measures. First Edition. Wiley, New York.

[2] Chistyakov, G. P. and Götze, F. (2004). Limit distributions of Studentized means. Ann.Probab. 32, 28-77.

[3] Csörgő M., Szyszkowicz B. and Wang Q. (2003). Donsker's theorem for selfnormalized partial sums processes. Annals of Probability 31, 1228-1240.

[4] Davydov, Y. A. (1970). The invariance principle for stationary processes. Theory of Probability and its Applications 15, 487-498.

[5] Dedecker, J., Merlevède, F. and Peligrad, M. (2009). Invariance principles for linear processes. Application to isotonic regression. To appear in Bernoulli. arXiv:0903.1951 
[6] de la Peña, V. and Giné, E. (1999). Decoupling. From dependence to independence. Springer.

[7] de la Peña, V., Lai, T. L. and Shao, Q. (2009). Self-Normalized Processes: Limit Theory and Statistical Applications. Springer, New York.

[8] Dickey, D.A. and Fuller W.A. (1979). Distribution of the estimators for autoregressive time series with a unit root. Journal of the American Statistical Association 74, 427-431.

[9] Dickey, D.A. and Fuller W.A. (1981). Likelihood ratio statistics for autoregressive time series with a unit root. Econometrica 49, 1057-1072.

[10] Feller, W. (1966) An Introduction to Probability Theory and Its Applications 2. Willey, New York.

[11] Giné, E., Götze, F. and Mason, D. M. (1997). When is the Student t statistic asymptotically standard normal?. Annals of Probability 25, 15141531.

[12] Knight, K. (1991). Limit theory for M-estimates in an integrated infinite variance process. Econometric Theory 7, 200-212.

[13] Kuelbs, J. (1985). The LIL when X is in the domain of attraction of a Gaussian Law. Annals of Probability 13, 825-859.

[14] Kulik, R. (2006). Limit theorems for self-normalized linear processes. Statistics and Probability Letters 76, 1947-1953.

[15] Lamperti, J. (1962). Semi-stable stochastic processes. Trans. Amer. Math. Soc. 104, 62-78.

[16] Mikosch, T. Gadrich, T. Kliippelberg C. and Adler, R. J. (1995). Parameter estimation for ARMA models with infinite variance innovations. Annals of Statistics 23, 305-326.

[17] Phillips, P. C. B. (1987). Time series regression with a unit root. Econometrica 55, 277-301.

[18] Phillips, P, C. B. and Solo, V. (1992). Asymptotics for linear processes. Ann. Statist. 20, 971-1001.

[19] Seneta E. (1976). Regularly Varying Functions. Lecture Notes in Mathematics 508, Springer.

[20] Sowell, F. (1990). The fractional unit root distribution. Econometrica 58, 495-505.

[21] Taqqu, M. S. (1975). Weak convergence to fractional Brownian motion and to the Rosenblatt process. Zeitschrift für Wahrscheinlichkeitstheorie und verwandte Gebiete 31, 287-302. 
[22] Wang, Q., Lin Y. X. and Gulati, C. M. (2003). Strong Approximation for Long Memory Processes with Applications. Journal of Theoretical Probability 16, 377-389.

[23] Wu, W. B. (2003). Additive functionals of infinite-variance moving averages. Statistica Sinica 13, 1259-1267.

[24] $\mathrm{Wu}, \mathrm{W}$. B. (2006). Unit root testing for functional of linear processes. Econometric Theory 22, 1-14.

[25] Wu, W. B. and Min, W. (2005). On Linear Processes with Dependent Innovations. Stochastic Processes and their Applications 115, 939-958. 